\title{
ENQUÊTE SUR LES SCIENCES
}

\author{
Jochen Hoock
}

Detlef Haberland, Hrsg., Engelbert Kaempfer (1651-1716). Ein Gelehrtenleben zwischen Tradition und Innovation. Wiesbaden, Harrassowitz, 2004. 15,5 × 23,5, 264 p.

Engelbert Kaempfer fut le premier japonologue européen. Le volume, qui précède l'édition critique de ses œuvres conservées au British Museum, retrace les étapes essentielles de sa vie et de ses travaux en les situant dans le contexte des découvertes de la fin du XVII ${ }^{\mathrm{e}}$ siècle. Les analyses concernent autant l'histoire culturelle des sociétés européennes et asiatiques que le développement des sciences de la vie au début du XVIII ${ }^{\mathrm{e}}$ siècle.

Andreas B. KILChER, Mathesis und poiesis. Die Enzyklopädik der Literatur, 1600-2000. Munich, Fink, 2003. 15,5 ×23,5, 536 p.

L'ouvrage s'intéresse aux rapports entre littérature et savoirs à l'époque moderne en revenant sur l'aventure encyclopédique et l'évolution des formes de savoir depuis le début du XVII ${ }^{\mathrm{e}}$ siècle. La lecture que propose Andreas B. Kilcher couvre l'ensemble de l'espace européen et intègre les réseaux de communication et d'édition comme la place du lecteur tout en proposant un vaste tableau d'une histoire des savoirs qui montre la place des belles-lettres dans l'évolution des sciences modernes.

Martin Carrier, Johannes Roggenhofer, Günter KüPPers et Philippe Blanchard, éd., Knowledge and the world. Challenges beyond the science wars. Berlin/New York, Springer, 2004. $15,5 \times 23,5,326 \mathrm{p}$.

Ce volume est issu d'un colloque du ZIF (Centre de recherches interdisciplinaires) de l'université de Bielefeld. Il aborde la controverse provoquée par Alan Sokal en essayant de la replacer dans le débat épistémologique depuis le début du $\mathrm{XX}^{\mathrm{e}}$ siècle. Il ne se borne pas seulement à retracer le débat mais aborde toutes les dimensions d'une controverse qui oppose une approche herméneutique au réalisme scientifique dont Sokal et Bricmont se font les défenseurs. C'est une contribution majeure aux débats actuels en histoire des sciences. 


\section{RELATIVITÉS}

Charles AlunNI

Erhard Scholz, éd., Hermann Weyl's Raum, Zeit, Materie and a general introduction to his scientific work. Bâle, Birkhäuser, 2001. $17 \times 24$, vI-403 p., ill. (DMV Seminar, Bd 30).

Cet ouvrage fera date, et il est voué à devenir canonique dans le champ des études contemporaines sur Hermann Weyl. Le volume est issu d'un séminaire de la Deutsche Mathematiker-Vereinigung qui s'est tenu du 24 au 31 mai 1992 : «We chose to concentrate the seminar on Hermann Weyl's contributions to the rise of general relativity and unified field theories. This topic allows, and in fact even demands, close cooperation and exchange between mathematics, philosophy, physics and their respective histories. Thus, in addition to its main goal, the seminar served to promote interchange across the boundaries of the disciplines involved, their respective histories, the general history of science and intellectual history $\gg(\mathrm{p} . \mathrm{v})$. Une attention toute particulière est portée aux changements de contenu et aux écarts de présentation entre les différentes éditions du traité de Weyl, entre 1918 et 1923 : «The background, context and extensions of the developments formed the pivot of the seminar $\gg(\mathrm{p} . \mathrm{v})$.

Indispensable vade-mecum du physico-mathématicien comme du philosophe qui ne peuvent ignorer la pensée de Weyl !

Vladimir P. VIZGIN, Unified field theories in the first third of the $X X^{\text {th }}$ century. Transl. from the Russian by Julian BARBOUR. Bâle, Birkhäuser, 1994. $17 \times 24$, XIX-335 p., bibliogr. (Science networks historical studies, 13).

Vladimir P. Vizgin propose une approche synthétique du programme ouvert par les théories physiques du champ unifié, lui-même issu de la théorie générale de la relativité. À la lumière de ce programme, la tâche fondamentale fut la recherche de schèmes géométriques plus généraux que la géométrie riemannienne, schèmes pouvant conduire à des équations du champ unifié (champ gravitationnel + champ électromagnétique) suffisamment non triviales pour inclure dans ses solutions les aspects corpusculaires et quantiques de la matière.

Le livre rend compte de ce programme, grandiose dans son inspiration, d'une synthèse géométrique du champ en physique. Il traite de ses premiers succès, des espoirs qu'il a pu susciter au début des années 1920, de ses difficultés, de ses échecs et des avancées vers de nouveaux développements jusqu'au début des années 1930, au moment où les succès de la théorie quantique, la découverte de nouvelles particules élémentaires et de leurs interactions poussèrent ce programme incomplet dans l'ombre, tout en lui opposant de nouveaux problèmes extrêmement sérieux.

Cet ouvrage de référence s'appuie évidemment sur les travaux de Tonnelat, mais également sur ceux de Pauli, Bergmann, Eddington, Weyl, Beck, Landé, Jordan, Schrödinger, Schmutzer, Rumer, Treder, Petrov et bien d'autres. 
Anadijiban DAs, The Special Theory of relativity. A mathematical exposition. New York, Springer, 1993. $16 \times 24$, XII- 214 p., ill., bibliogr., index (Universitext).

Cet ouvrage présente la théorie de la relativité restreinte du point de vue de sa structure axiomatique : de l'espace vectoriel minkowskien quadridimensionnel aux champs classiques dans l'espace de phase (covariant) ou étendu à huit dimensions (espace de Born-Yukawa), en passant par l'espace des spineurs et les champs de jauge non-abélienne. Il s'agit d'une remarquable interface entre mathématiques abstraites et physique théorique.

José LeITe Lopes, Théorie relativiste de la gravitation. Paris, Masson, 1993. $16 \times 24$, XII- 224 p., ill., bibliogr., index (Physique fondamentale et appliquée).

Présentation remarquablement condensée des bases de la théorie, et en particulier des groupes de Lorentz et de Poincaré. Lopes met aussi en évidence les propriétés de jauge des champs de gravitation. Philosophiquement très suggestif.

Hans C. OHANiAn et Remo RufFini, Gravitation and spacetime. $2^{\mathrm{e}}$ éd., New York/Londres, W. W. Norton, 1994. $16 \times 24$, XV-679 p., ill., bibliogr., index.

De la première édition (1976), écrite sans la collaboration de Remo Ruffini, John Wheeler affirmait déjà qu'il s'agissait du «meilleur livre présent sur le marché de près de 500 pages traitant de la gravitation et de la relativité générale ». Cette nouvelle version, entièrement refondue, donne, comme la première, une place considérable à la description des résultats expérimentaux, mais l'un des intérêts de cette réédition tient en particulier dans une initiation au formalisme des formes différentielles et du calcul extérieur d'Élie Cartan (nouvelles sections ajoutées aux chapitres II et VI). Nombre de notes et commentaires sur l'histoire et les limites du passage entre ce formalisme et le calcul tensoriel ordinaire sont suffisamment rares dans la littérature (relativiste ou mathématique) pour que cela soit souligné. Le philosophe « flambeur » saura s’y délecter...

Richard P. FEYNMAn, Feynman lectures on gravitation. Ed. by Brian HATFIELD, foreword by John Preskill and Kip S. Thorne. Reading (MA)/Menlo Park (CA)/Paris, AddisonWesley Publishing, 1995. $16 \times 24$, XL-232 p., ill., bibliogr., index.

Cet ouvrage reprend les vingt-sept cours de Richard P. Feynman au Caltech (California Institute of technology) entre 1962 et 1963. L'approche non-traditionnelle du sujet nous renseigne sur la manière d'aborder la gravitation de Feynman. Ce texte est peu indiqué pour des débutants. Le point de vue dogmatiquement non-géométrique qui trouve les faveurs de Feynman est présenté de manière plus systématique et compréhensive par Steven Weinberg. Il est ici fondé sur un intérêt primordial pour les aspects quantiques de la gravité. Ces notes s'accompagnent de digressions fascinantes sur les fondements de la physique. Pour tous les admirateurs du grand style Feynman. 
Markus KRIELE, Spacetime. Foundations of general relativity and differential geometry. Berlin/New York, Springer, 1999. $16 \times 24$, XVIII-436 p., bibliogr., index (Lecture Notes in Physics, m 59).

Markus Kriele nous livre ici une introduction à la géométrie pseudo-riemannienne orientée sur les concepts géométriques. Elle s'adresse aux mathématiciens et physiciens-mathématiciens, mais sur la base d'une justification physique du cadre mathématique. La plupart des textes modernes présentent aux mathématiciens la géométrie pseudo-riemannienne (et la relativité générale) sans articulation de cette géométrie aux propriétés de l'espace-temps, la développant plutôt comme une théorie mathématique purement abstraite. Contre ces approches trop formelles, et «[t]o ensure that the mathematical description mirrors the physical concepts, all definitions have a justification in this book. This approach also leads to a careful treatment of the structural aspects of the mathematics » (p. IX).

Cet ouvrage, extrêmement riche et complet, reconnaît sa dette «philosophique » du côté d'Hermann Weyl et de Jürgen Ehlers. Il faut noter à nouveau la grande qualité dans la facture de l'ouvrage, et en particulier l'usage inédit de marginalia que l'on qualifierait volontiers d' " ascenceurs-guides » fournissant des raccourcis : en réalité, les matériaux sont ordonnancés de manière à ce que le lecteur puisse utiliser le texte comme source de références.

Gerard't HoofT, Introduction to general relativity. Cours en ligne, version du 8/4/2002, 59 p., téléchargeables au format ps sur http://www.phys.uu.nl/ thooft/

Élève de Martin Veltman (Institute for Theoretical Physics de l'université d'Utrecht), Gerard't Hooft est devenu l'un des plus éminents spécialistes des théories de jauge et de la renormalisation de la «théorie pure » de Yang-Mills (celle dont tous les champs sont sans masse). "General relativity is a beautiful scheme for describing the gravitational field and the equations it obeys. Nowadays this theory is often used as a prototype for other, more intricate constructions to describe forces between elementary particles or other branches of fundamental physics. This is why in an introduction to general relativity it is of importance to separate as clearly as possible the various ingredients that together give shape to this paradigm » (p. 2).

John Earman, Michel Janssen et John D. Norton, éd., The Attraction of gravitation. New studies in the history of general relativity. Boston/Bâle/Berlin, Birkhäuser, 1993. $16 \times 24$, X-432 p., bibliogr., index (Einstein Studies, vol. V).

Les « Einstein Studies », dirigées par Don Howard et John Stachel et publiées sous l'égide du Center for Einstein Studies de l'université de Boston, se présentent comme des « séries » multidisciplinaires visant à refléter la grande variété des contributions einsteiniennes au $\mathrm{XX}^{\mathrm{e}}$ siècle (et au XXI ${ }^{\mathrm{e}}$ !). L'un des buts principaux de cette collection est l'établissement et le maintien de hauts standards pour l'étude et la recherche concernant la vie, l'œuvre et l'époque d'Einstein. Elle consiste essentiellement en la publication de congrès ou de colloques consacrés à Einstein : Osgood Hill Conference (1986) pour le volume I ; Luminy (1988) pour le volume III ; Pittsburgh at Johnstown, Pennsylvanie (1991) pour le volume V. Incontournables pour un contact permanent et actualisé avec les études einsteiniennes. 
Evert Jan Post, Formal Structure of electromagnetics. General covariance and electromagnetics, rééd. Mineola (NY), Dover Publications, 1997. $13 \times 21,5$, XIV-204 p., ill., bibliogr., index.

La Structure formelle de l'électromagnétisme, dont la première édition date de 1962 (Amsterdam, North-Holland), est l'œuvre assez singulière du physicien hollandais Evert Jan Post. Cet élève de J. A. Schouten et de D. Van Dantzig doit beaucoup à leur approche de l'électromagnétisme, et il en propose ici une réactivation à partir du principe philosophique suivant : "Physics is badly in need of principles which help to single out the physically most significant structures among the multitude of mathematical possibilities which are admissible for the description of physical phenomena. In other words, to bridge the gap between physics and its mathematical image one may attempt to tighten the rules for expressing physics in terms of mathematics. We shall endeavour to do so, guided by principles of a knowledge-theoretical nature »(p.1). En ce sens, Post révise et complète un certain nombre de principes généraux sous-tendant la plus ancienne des théories du champ, à savoir la théorie du champ électromagnétique. Le noyau conceptuel de ce travail tourne autour de la réaffirmation forte du principe de covariance générale; l'ouvrage est (entre autres) passionnant pour son analyse raffinée de ce principe, pour la reconstruction de son évolution historique et pour sa tentative d' "émancipation». Dans un article intitulé «A dutch uncle tirade about relativity matters » (téléchargeable sur son site), l'auteur dirige ses critiques à la fois contre l'establishment pour avoir silencieusement accepté une situation d' « émasculation » de fait du principe de covariance générale, et en direction des galiléens qui ont pris les positions négatives de cet establishment trop au sérieux. Une analyse raffinée et de nouveaux développements du principe « resolve many problems of the Galileans as well as the establishment predicament of failing to reconcile quantum theory and relativity ». Or, les graines pour de tels développements étaient déjà semées dans les années 1920 et 1930 ; et si l'auteur (comme ici) les présente, c'est « to be preserved for the posterity ».

Les tableaux du chapitre I, «Principle of covariance », sont extrêmement précieux. Le reste de l'ouvrage fait appel aux ressources notationnelles de Schouten qui n'étaient rien de moins qu'un affreux « cauchemar » pour Hermann Weyl. C'est une lecture à la fois nécessaire pour l'étude (ardue) de la postérité de l'école hollandaise de géométrie différentielle, et passionnante pour déchiffrer de réels enjeux théoriques. Sur ce point, l'ouvrage ne doit pas être déconnecté des essais plus «philosophiques » de Post.

Laurent NotTALE, L'Univers et la lumière. Cosmologie classique et mirages gravitationnels. Paris, Flammarion, 1994. $16 \times 24,288$ p., index (Nouvelle bibliothèque scientifique).

Ce livre, réédité en 1997 (Paris, Flammarion, « Champs, 383 »), qui est le récit rigoureux et passionnant de la rencontre entre la cosmologie classique et les « mirages gravitationnels », découvre pour le lecteur tous les concepts fondamentaux. Particulièrement impressionnant se révèle le traitement très clair de la constitution (et du sens) du fameux «tenseur d'Einstein » : y sont expliqués, de manière extrêmement limpide, le mécanisme et l'importance de sa «divergence nulle ». L'auteur a reçu pour cet ouvrage le prix du livre d'Astronomie en 1995. 
Laurent NotTale, La Relativité dans tous ses états. Au-delà de l'espace-temps. Paris, Hachette Littératures, 1998. $16 \times 24,319$ p.

C'est là un essai (2e éd. Paris, Hachette Littératures, 2000) à la fois très accessible et profondément novateur : ces deux qualités, fort rares aujourd'hui, sont rendues possibles par l'extrême maitrise et profondeur de la pensée einsteinienne et de ses ultimes conséquences conceptuelles. Laurent Nottale, entre autres, illustre bien le principe relativiste fondamental : «Au commencement était la Relation» (Gaston Bachelard, La Valeur inductive de la Relativité, Paris, Vrin, 1929). Cet aphorisme, simple d'apparence, est ce qu'il y a de plus difficile à accepter et à intégrer dans nos cadres de pensée. Ce devrait être l'objet de plusieurs congrès de philosophes !

Plus qu'une théorie particulière, la démarche de Nottale est celle d'une ouverture d'horizon comparable aux effets heuristiques de la conceptualisation, il y a près d'un siècle, de l'espacetemps dans le cadre de la relativité restreinte. La qualité de cette théorie réside précisément dans cette puissance qu'il faut explorer. En autorisant une démarche de pensée, un protocole d'induction au sens de Bachelard, la théorie ne reste pas seulement programmatique : ses résultats fascinants sur les exoplanètes, ses développements récents sur les champs commandés par les équations de Schrödinger et de Dirac en donnent déjà les preuves.

Nottale prend Einstein au mot, radicalise la « relativation » en l'appliquant aux principes fondamentaux de la physique «relativiste » inaugurés par le geste galiléen, ce qui ne manque pas de résonner sur la question du concors discors entre Relativité et Quantique. C'est par ailleurs une théorie promise à des développements en biologie. Une mine d'investigations pour le philosophe... (Pour consulter les résultats et développements récents, voir : http://www.daec.obspm.fr/users/nottale/fr.menure.htm)

James J. Callahan, The Geometry of spacetime. An introduction to special and general relativity. New York, Springer 2000. $18 \times 24$, XVI- 451 p., bibliogr. (Undergraduate texts in mathematics).

L'idée de cet ouvrage plante ses racines dans une série de trois conférences tenues par John Milnor à l'université de Warwick au printemps 1978. Il s'agit d'une approche géométrique particulièrement claire, et dont la lisibilité doit beaucoup à l'usage systématique de « diagrammes » (dont certains en marge, ce qui s'avère extrêmement esthétique et efficace) particulièrement soignés et « épurés ».

Angelo Genovesi, Il Carteggio tra Albert Einstein ed Edouard Guillaume. «Tempo universale » e teoria della relatività ristretta nella filosofia francese contemporanea. Milan, Franco Angeli, 2000. $16 \times 23,174$ p., bibliogr., index (Filosofia).

Cet ouvrage, que l'on doit à l'école italienne (et plus précisément à l'école pisane) d'histoire et de philosophie des sciences, offre une approche totalement nouvelle de l'élaboration des « idées » et de la réception françaises de la relativité einsteinienne.

Le livre se divise en deux parties. La première s'intitule " "Temps universel" et théorie de la relativité restreinte dans la philosophie française contemporaine »; la seconde a pour titre « Albert Einstein, Édouard Guillaume, Lettres, 1917-1948». Il s'agit ici (p. 81-170) de la transcription et de l'édition tout à fait neuve de quatorze lettres d'Einstein, et de seize de 
Guillaume conservées aux Albert Einstein Archives de la Jewish National \& University Library de Jérusalem. Au premier regard, ces lettres ont un aspect très technique que reflète la présence massive d'équations, de figures et de diagrammes. Question (qui nous renvoie à la première partie de l'ouvrage) : qui est donc ce «M. Édouard Guillaume de Neuchâtel, Suisse »- et quel peut bien être l'enjeu philosophique d'une telle correspondance ?

Si le nom d'Édouard Guillaume est totalement absent de la biographie canonique d'Abraham Païs, on ne le rencontre dans l'édition française des Euvres choisies (Paris, Seuil, 1993) que dans la parenthèse d'une note (vol. III, p. 196, n. 3) consacrée à l'article de vulgarisation d'Einstein (1910) traduit par Édouard Guillaume et paru dans les Archives des sciences physiques et naturelles de Genève sous le titre «Le principe de relativité et ses conséquences dans la physique moderne ».

En réalité, Édouard Guillaume, né à Les Verrières dans le canton de Neuchâtel en 1883 (mort à Delémont, canton du Jura, en 1959), n'est autre que le cousin du prix Nobel de physique (1920), directeur de l'Office international des poids et des mesures situé à Sèvres, Charles-Édouard du même nom. Notre Édouard obtient, quant à lui, le grade de docteur en philosophie (section scientifique) en 1908 à l'université de Zurich, avec une thèse portant sur Les Phénomènes de Bose et les lois de l'électrisation de contact. Surtout, il occupe la même année le poste de technicien expert de deuxième classe au Bureau de la propriété intellectuelle de Berne où il devient le collègue d'Einstein.

Plus important pour la suite, Einstein connaissait bien Édouard Guillaume en tant qu'éditeur d'Henri Poincaré. Guillaume se chargea en effet de la réédition des célèbres mémoires publiés sous le titre La Mécanique nouvelle. Conférence, mémoire et note sur la théorie de la relativité (Paris, Gauthier-Villars, 1924). Guillaume en assura l'introduction qui représente sa dernière intervention publique concernant la nouvelle configuration de l'espace-temps proposée par la théorie einsteinienne : question qui avait concentré l'intérêt du physicien et mathématicien helvétique depuis sa traduction du mémoire d'Einstein en 1910. Ce qu'a alors en tête le physicien suisse donne l'équation fondamentale de ses positions ; positions qui détermineront toute la scène philosophique française sur la relativité. Et ce que prouve ad libitum et définitivement la reconstitution rigoureuse conduite par Angelo Genovesi, c'est que l'entreprise néo-poincaréenne de Guillaume se présente comme le réservoir central auquel viendront puiser toutes les opérations françaises de déligitimation « conventionaliste » des théories de la relativité d'Einstein : de la falsification historique de Lucien-Fabre («cette canaille », dixit Maurice Solovine à Einstein, lettre du 20 juin 1921), au « réalisme» opportuniste et positiviste de Paul Dupont (La Notion de temps d'après Einstein, Paris, Alcan, 1921), en passant par le «scepticisme» de Marcel Brillouin, les « conservatismes » de Georges Sagnac (« un nouveau Poincaré », d'après Fabre) et de Henri Varcollier, l'opposition mathématicienne de Paul Painlevé et d'Émile Picard, et, last but not least, la grande entreprise bergsonienne de Durée et simultanéité (Paris, Alcan, 1922) qui renvoie à Guillaume au chapitre v.

Tout cela est parfaitement mis en lumière par Genovesi dans son introduction. L'enjeu est double : 1) c'est évidemment la question fondamentale du «temps » (et du déclin des absolus) qui se décide ici ; 2) c'est, parallèlement à la délégitimation « néo-conventionaliste » (et «positiviste») des théories d'Einstein, la fameuse question de la priorité-paternité poincaréenne de la relativité qui s'y noue. L'entreprise sera radicalisée en ce sens en 1953 par Edmund Whittaker dans son History of the theories of aether and electricity (Londres/ Édimbourg/Paris, Nelson and Sons), vol. II, The Modern Theories 1900-1926, au chapitre : « The relativity theory of Poincaré and Lorentz. » À peine cinq ans plus tôt, Guillaume n'écrivait-il pas à Louis de Broglie, en référence à sa réédition des mémoires de Poincaré : « Je me suis permis d'y ajouter une Introduction où, par un diagramme, je confirme les vues de 
Poincaré. Le travail du grand géomètre contient déjà la cinématique d'Einstein » (lettre du 9 mars 1948). On sait qu'à la différence du débat des priorités Hilbert-Einstein concernant la relativité générale (débat définitivement tranché par les récents travaux de Corry-RennStachel), celui-là ne l'est toujours pas !

Parce que la question du temps demeure « la croix du philosophe » (dixit Husserl), et que la relativité d'Einstein est une clef essentielle à l'élucidation de cette cruci-fiction, la mise au point de Genovesi constitue une véritable propédeutique pour le travail du philosophe. 